\title{
ERZIEHUNGS- UND JUGENDFRAGEN: DIE BANDE ENGER GESTALTEN UND DAS VERSTÄNDNIS FÜREINANDER VERTIEFEN?
}

In einem Vermerk der französischen Botschaft in Bonn vom 19. Januar 1963' wurde bereits drei Tage vor der Unterzeichnung des Élysée-Vertrages festgestellt, da $\beta$ die engen transatlantischen Bindungen der Bundesrepublik der künftigen deutsch-französischen Kooperation zumindest im politischen und militärischen Bereich im Weg stehen könnten. Und auch auf deutscher Seite rechnete man damit, daß die Unterzeichnung einer deutsch-französischen Erklärung größere politische Probleme mit sich bringen könnte - dies zeigen verschiedene Stellungnahmen des Auswärtigen Amtes. ${ }^{2}$

Man kann also davon ausgehen, daß sich beide Seiten bei der Unterzeichnung des Vertrages durchaus der Schwierigkeiten bewußt waren, die sich im politisch-diplomatischen und im militärischen Bereich ergeben könnten. Allerdings hatte der Vertrag darüber hinaus einen sehr viel weitergehenden Anspruch - so wurde bereits in der vorangestellten Gemeinsamen Erklärung betont, da $₫$ »die Versöhnung zwischen dem deutschen und dem französischen Volk, die eine Jahrhunderte alte Rivalität beendet, ein geschichtliches Ereignis darstellt, das das Verhältnis der beiden Völker zueinander von Grund auf neu gestaltet ${ }^{3}$ Ein wirklich großes Ziel für einen politischen Vertrag!

Unter dieser langfristigen Perspektive fällt dem mit »Erziehungs- und Jugendfragen« überschriebenen Teil $\mathrm{C}$ ein besonderes Gewicht zu: Die bestehenden Bande zwischen den Völkern sollten enger gestaltet und das Verständnis füreinander vertieft werden.

Ich möchte zunächst einen kurzen Blick auf die Rahmenbedingungen für die auswärtige Kulturpolitik zwischen den beiden Staaten werfen, vor deren Hintergrund die entsprechenden Bestimmungen zu sehen sind und dann auf die einzelnen in diesem Abschnitt erwähnten Punkte eingehen.

MAE/Paris, Europe 1961-1970, RFA, Bd. 1600.

2 So insbesondere in der Aufzeichnung von Ministerialdirektor Jansen vom 20.1.1963, in: Akten zur Auswärtigen Politik der Bundesrepublik Deutschland (AAPD) 1963, Bd. 1, Dok. 35, S. 109.

3 Gemeinsame Erklärung und Vertrag über die deutsch-französische Zusammenarbeit vom 22.1.1963, in: Bundesgesetzblatt 1963, Teil II, S. 705-710, hier S. 705. 


\section{Rahmenbedingungen der deutschen und französischen auswärtigen Kulturpolitik}

Für Frankreich hatten die weltpolitischen Entwicklungen in den 1950er Jahren große Umbrüche mit sich gebracht: Angesichts der Entkolonialisierung, des verlorenen Indochina-Kriegs, des Ausgangs der Suez-Krise und der Blockbildung im Zeichen des Kalten Kriegs, nicht zuletzt auch angesichts des wirtschaftlichen Aufstiegs des deutschen Nachbarn mußte man in Paris schmerzlich erkennen, daß Frankreich eben keine Weltmacht mehr war. Die französische Politik versuchte dieser Entwicklung unter anderem dadurch entgegenzutreten, daß ein neuer Schwerpunkt auf die auswärtige Kulturpolitik gelegt wurde. ${ }^{4} \mathrm{Zu}$ diesem Zweck wurde die "Direction Générale des Relations Culturelles" (DGRC) im Quai d'Orsay 1956 komplett umstrukturiert und dabei in "Direction Générale des Affaires Culturelles et Techniques" (DGAT) umbenannt. ${ }^{5}$ Am 31. Juli 1957 wurde Außenminister Christian Pineau mit der Bildung eines Komitees zur Ausarbeitung eines Fünfjahresplans beauftragt, das die Zielsetzungen der künftigen kulturpolitischen Aktivitäten im Ausland festlegen sollte. ${ }^{6}$ Das Komitee ${ }^{7}$ erstellte Ende Juni 1958 einen Abschlußbericht, den der neue Außenminister Couve de Murville am 10. September 1958 dem Kabinett vorlegte; die neue Regierung verabschiedete daraufhin den Fünfjahresplan (»Premier programme quinquennal d'expansion et de reconversion des activités culturelles françaises à l'étranger «), ${ }^{8}$ der sich auf die Jahre 1959 bis 1963 bezog. ${ }^{9}$ Er war somit quasi ein Erbe der IV. Republik, welches nun in der V. Republik unter Général de Gaulle Anwendung finden sollte.

Auf bundesdeutscher Seite gab es zwar seit 1949 diverse Ansätze zu einer staatlich geförderten auswärtigen Kulturpolitik, ${ }^{10}$ insgesamt spielten kulturelle Aspekte in der bundesdeutschen Außenpolitik zunächst aber eher eine be-

4 Eine ähnliche Neuakzentuierung der französischen Außenpolitik ließ sich auch nach 1871 beobachten; vgl. Hélène Trova, Le statut juridique de l'action culturelle et linguistique de la France à l'étranger, Paris 1994, S. 30f;; François ROCHE, Bernard PINIAU, Histoire de diplomatie culturelle des origines à 1995, Paris 1995, S. 14f.

5 TROVA (Anm. 4), S. 36f.; Victoria ZNINED-BRAND, Deutsche und französische auswärtige Kulturpolitik. Eine vergleichende Analyse, Frankfurt a. M. 1999, S. 46.

- RoChe, Piniau (Anm. 4), S. $91 \mathrm{f}$.

7 Zusammensetzung: TROVA (Anm. 4), S. 37.

8 RoChe, PINIAU (Anm. 4), S. 92.

9 Ansbert BAUMANN, Begegnung der Völker? Der Élysée-Vertrag und die Bundesrepublik Deutschland. Deutsch-französische Kulturpolitik von 1963 bis 1969, Frankfurt a. M. 2003, S. 93.

10 Hans ARNOLD, Auswärtige Kulturpolitik. Ein Überblick aus deutscher Sicht, München, Wien 1980, S. 14. 
scheidene Rolle." Immerhin wurden ab Mitte der 1950er Jahre kulturpolitische Vereinbarungen mit anderen Staaten abgeschlossen - das erste schriftlich fixierte Kulturabkommen im übrigen am 23. Oktober 1954 mit Frankreich. ${ }^{12}$ Allerdings handelte es sich bei diesen Abkommen eher um Absichtserklärungen $^{13}$ - die Kulturpolitik fällt in der Bundesrepublik bekanntlich unter die Hoheitsrechte der Länder, so daß die Bundesregierung hier rechtlich gebunden war. ${ }^{14}$ Dennoch gewann die auswärtige Kulturpolitik Ende der 1950er Jahre deutlich an Bedeutung; dies zeigte sich nicht zuletzt am Ausbau der Kulturabteilung des Auswärtigen Amtes, welche ab 1958 mit einem deutlich höheren Budget ${ }^{15}$ ausgestattet wurde und ab 1959 unter der Leitung von Ministerialdirektor Dieter Sattler mehr und mehr an Einfluß gewann. ${ }^{16}$

Man kann also festhalten, daß Anfang der 1960er Jahre sowohl auf bundesdeutscher als auch auf französischer Seite ein verstärktes Interesse an der auswärtigen Kulturpolitik zu verzeichnen war. Allerdings zeigte sich im Frühjahr 1962, da B gerade in den deutsch-französischen Beziehungen in dieser Hinsicht Wunschdenken und Realität auseinanderklafften: Aus diesem Grund forderte die französische Botschaft in Bonn dringend eine Intensivierung der französischen Kulturpolitik in Deutschland, ${ }^{17}$ und der Quai d'Orsay kündigte daraufhin tatsächlich eine "reconversion de notre action culturelle en Allemagne ${ }^{18}$ an.

Auch auf deutscher Seite war man mit der Entwicklung unzufrieden. So konstatierte der deutsche Botschafter Herbert Blankenhorn, daß ein verstärktes kulturpolitisches Engagement der Bundesrepublik in Frankreich im Interesse einer "Stabilisierung des deutsch-französischen Verhältnisses unaufschiebbar ${ }^{19}$ sei. Interessanterweise wurde von Seiten der deutschen Diplomaten mehrfach versucht, diesem Argument unter Hinweis auf die kulturpolitischen Aktivitäten der

"BAUMANN (Anm. 9), S. 94.

12 Horst MölLER, Klaus HILDEBRAND (Hg.), Die Bundesrepublik Deutschland und Frankreich. Dokumente 1949-1963 (BDFD), Bd. 1, München 1997, Nr. 40, S. 184-188. Mit den USA war bereits am 9.4.1953 eine kulturpolitische Vereinbanung in Form eines Notenwechsels geschlossen worden.

13 Christoph HIRSCH, Kulturhoheit und Auswärtige Gewalt, Berlin 1968, S. 88.

14 BaUmanN (Anm. 9), S. 111-128.

is Manfred ABELEIN, Die Kulturpolitik des Deutschen Reiches und der Bundesrepublik Deutschland. Thre verfassungsgeschichtliche Entwicklung und ihre verfassungsrechtlichen Probleme, Köln, Opladen 1968, S. 161; Dieter SATTLER, Die dritte Bühne der AuBenpolitik, in: Berthold MARTIN (Hg.), Jahrbuch der auswärtigen Kulturbeziehungen 1964, Bonn 1964, S. 13-21.

16 ZNINED-BRAND (Anm. 5), S. 43.

17 Französische Botschaft in Bonn (Service culturel) an Außenminister Couve de Murville, 5.1.1962; MAE, Europe 1961-1970, RFA, Bd. 1475.

18 Note du Ministère des Affaires étrangères, 9.2.1962; MAE, Europe 1961-1970, RFA, Bd. 1475.

19 Deutsche Botschaft, Paris, Vermerk, 11.1.1962, betr. Ausweitung der kulturellen Arbeit in Frankreich; PA/AA, B 90-600, Bd. 545. 
DDR in Frankreich politisch eine gewisse Nachdrücklichkeit zu verleihen, so beispielsweise mit der Feststellung, die intellektuellen Kreise in Frankreich seien alle wvon der rein politischen Kulturpropaganda der Sowjet-Zone mehr oder weniger beeinflußt ${ }^{20}{ }^{20}$ Der im Frühsommer 1962 begonnene Bau eines großen bundesdeutschen Kulturinstituts in Paris machte deutlich, daß die Bonner Regierung von den vielfältigen Argumenten nicht unbeeindruckt war und ganz offensichtlich neue kulturpolitische Akzente setzen wollte. ${ }^{21}$

Die politische Annäherung, die sich im Anschluß an Adenauers Frankreichbesuch vom Juli 1962 vollzog, rückte die Kulturpolitik noch mehr ins Blickfeld der politischen Akteure. Unmittelbar vor seinem Gegenbesuch in der Bundesrepublik im September 1962 kündigte de Gaulle daher an, daß er neben der politischen und militärischen auch eine enge Zusammenarbeit im kulturellen und wissenschaftlichen Bereich anstrebe. ${ }^{22}$ Erziehungsminister Pierre Sudreau erstellte daraufhin einen Vermerk, in welchem er diesbezüglich konkrete Vorschläge lieferte. ${ }^{23}$ Bei ihren Gesprächen waren sich de Gaulle und Adenauer darin einig, da $B$ in diesen Fragen ebenfalls Fortschritte erzielt werden sollten, ${ }^{24}$ und die "Erziehungs- und Jugendfragen « tauchten folglich auch im Memorandum der französischen Regierung vom 19. September $1962^{25}$ auf. Die deutsche Reaktion im Antwortmemorandum vom 8. November $1962^{26}$ war zwar recht positiv, jedoch wurde in einem Nebensatz auf ein wesentliches Problem hingewiesen, nämlich auf die Zuständigkeit der Länderregierungen im Bereich des Erziehungswesens. $^{27}$

Diese Komplikation auf deutscher Seite erwies sich während der darauffolgenden Wochen als beträchtlicher Hemmschuh: Bei der Ausarbeitung der Entwürfe für die beabsichtigte deutsch-französische Erklärung wurde mehr und mehr klar, daß das Problem der Beteiligung der Länder in angemessener

${ }^{20}$ Vermerk des Auswärtigen Amtes, 18.4.1962: Ergebnisse der Arbeitstagung des GoetheInstituts in Paris vom 11.-13.4.1962; PA/AA, B 90-600, Bd. 518.

21 Vermerk des Auswärtigen Amtes, 20.6.1962; ibid.

22 Knut LINSEL, Charles de Gaulle und Deutschland 1914-1969, Sigmaringen 1998, S. $200 f$.

${ }^{23}$ Le Ministre de l'Education nationale, Note à l'attention du ministre des Affaires étrangères, 1.9.1962; MAE, Europe 1961-1965, RFA, Bd. 1578 (alte Signatur).

24 Konrad ADENAUER, Erinnerungen 1959-1963. Fragmente, Stuttgart 1968, S. 179f.

25 MAE, Europe 1961-1970, RFA, Bd. 1598; deutsche Übersetzung: PAAA, B 90-600, Bd. 411; Hauptstaatsarchiv Stuttgart (HStASt), EA 3/505, Nr. 327/1, Blatt 12 - Anlage; Hauptstaatsarchiv Düsseldorf (HStAD), Kultusministerium NW 69-314, Bl. 39.

${ }^{26}$ Memorandum der Bundesregierung vom 8.11.1962; PA/AA, B 90-600, Bd. 411; HStASt, EA 3/505, Nr. 327/1, Bl. 12 - Anlage; französische Übersetzung: MAE, Europe 19611965, RFA, Bd. 1598.

$27 \gg$ Der Vorschlag [...] wird von der Bundesregierung in Verbindung mit den Länderregierungen geprüft, die für Maßnahmen auf dem Gebiet des Erziehungswesens in der Bundesrepublik zuständig sind«; ibid. 
Weise berücksichtigt werden mußte, damit die kulturpolitischen Absichten überhaupt eine gewisse Effizienz haben könnten. ${ }^{28}$

Tatsächlich wurde dann im Élysée-Vertrag versucht, eine Lösung für dieses innerdeutsche Problem zu finden, indem bei den vorgesehenen Konsultationen eine nicht näher definierte "Persönlichkeit" als Gesprächspartner des französischen Erziehungsministers »benannt" werden sollte; diese "Benennung" sollte in Abstimmung mit den Länderregierungen erfolgen. ${ }^{29}$

Die Streitigkeiten zwischen Bund und Ländern über die Kompetenzen jener Institution zogen sich sechs Jahre lang hin, ehe schließlich im Februar 1969 eine Durchführungsvereinbarung zum Élysée-Vertrag unterzeichnet werden konnte, in der auch diese Frage endgültig geregelt wurde. ${ }^{30}$ In Gestalt des Kulturbevollmächtigten, den die Ministerpräsidenten aus ihren Reihen wählen, besitzt die Bundesrepublik nunmehr gegenüber Frankreich - und nur gegenüber Frankreich - einen »Bundeskulturminister«. Dies war die entscheidende Grundlage dafür, $\mathrm{da} ß$ auch kulturpolitische Ziele Gegenstand der vertraglich geregelten politischen Konsultationen sein konnten. Der Élysée-Vertrag ist also bis zum heutigen Tag der einzige völkerrechtliche »Kultur-Vertrag» der Bundesrepublik Deutschland der Versuch, die innerdeutsche Zuständigkeitsproblematik konsequent zu regeln, blieb einmalig und ist ein häufig verkannter großer Erfolg des Élysée-Vertrages.

\section{Die inhaltlichen Aspekte des Vertrages}

\section{Fremdsprachenunterricht}

Wenden wir uns nun den einzelnen Bestimmungen des mit "Erziehungs- und Jugendfragen « überschriebenen Absatzes zu: Die als erstes Ziel anvisierte Förderung des Fremdsprachenunterrichts stellt für die französische Politik bekanntermaßen ein Schwerpunkt dar, ${ }^{31}$ während dieses Thema für die bundesdeutsche Außenpolitik in den 1960er Jahren eher eine untergeordnete Rolle

28

29

Martin J. SATTLER, Der deutsch-französische Zusammenarbeitsvertrag. Eine Untersuchung zur Vertragsmacht des Bundes und der Länder, Meisenheim 1976, S. 6f. Die Verwendung des Begriffs »Benennung " weicht von der üblichen Terminologie internationaler Verträge ab und trägt der rechtlich komplizjerten Situation bewußt Rechnung; (Vermerk des Staatsministeriums Baden-Württemberg über die Stellung des Beauftragten der Bundesrepublik Deutschland für kulturelle Angelegenheiten im Rahmen des deutsch-französischen Vertrages yom 22.1.1963, S. 4f.; HStASt, EA 3/505, Nr. 327/2).

30 BaUMANN (Anm. 9), S. 141-181.

"Suzanne BALOUS, L'action culturelle de la France dans le monde, Paris 1970, S. 17. 
spielte. ${ }^{32}$ Hinzu kam das bereits erwähnte Kompetenzproblem mit den Bundesländern, die allein für den schulischen Sprachunterricht zuständig sind.

In diesem Zusammenhang ist bemerkenswert, daß das Französische an den höheren Schulen in Deutschland bis zum Jahr 1937 die erste unterrichtete lebende Fremdsprache war - erst unter den Nationalsozialisten wurde der Französischunterricht aus ideologischen Gründen zugunsten des Englischen an den Rand gedrängt. ${ }^{33}$ Nach 1945 erhielt Französisch dann lediglich in der französischen Besatzungszone wieder den Rang der ersten lebenden Fremdsprache. ${ }^{34}$ Im übrigen Gebiet der späteren Bundesrepublik hatte die britische oder die amerikanische Besatzungsmacht den weiteren Ausbau des Englischunterrichts begünstigt. ${ }^{35}$ Nach der Gründung der Bundesrepublik war somit in allen Ländern, mit Ausnahme von Baden, Rheinland-Pfalz und Württemberg-Hohenzollern, Englisch erste Fremdsprache; ein weiteres Zurückdrängen des Französischen schien daher nur noch eine Frage der Zeit zu sein. Frankreich versuchte zwar durch verschiedene Interventionen bei den Länderregierungen die Situation des Französischunterrichts insgesamt $\mathrm{zu}$ verbessern, konnte dabei jedoch keine entscheidenden Fortschritte erzielen. ${ }^{36}$ Die Frage spielte deswegen bei den schon Anfang der 1950er Jahre aufgenommenen Verhandlungen über ein deutsch-französisches Kulturabkommen eine wichtige Rolle; ${ }^{37}$ die französische Regienung konnte allerdings auch hier zu keinem größeren Erfolg kommen. Schließlich einigte man sich im Deutsch-Französischen Kulturabkommen vom 23. Oktober 1954 auf eine Kompromißformel, derzufolge die Vertragspartner lediglich "soweit irgend möglich Sorge dafür« tragen sollten, daß ein entsprechender »Unterricht $[\ldots]$ veranstaltet $[\ldots]$ wird . $^{38}$

Diese Absichtserklänung ließ, wie sich schon wenige Wochen später zeigen sollte, sehr viele Spielräume offen: Am 17. Februar 1955 unterzeichneten die Mi-

32 Ulrich AMMON, Zur Geschichte der Sprachverbreitungspolitik der Bundesrepublik Deutschland von den Anfängen bis 1985. Kommentierte Dokumentation der Diskussion im Bundestag mit anschließendem Ausblick, in: Deutsche Sprache, 17 (1989), S. 229263, hier S. 229f.; DERS., Die internationale Stellung der deutschen Sprache, Berlin, New York 1991, S. 524f.

33 Ansbert BAUMANN, Der sprachlose Partner. Das Memorandum vom 19. September 1962 und das Scheitern der französischen Sprachenpolitik in der Bundesrepublik Deutschland, in: Revue d'Allemagne et des pays de langue allemande, 34 (2002) 1, S. 55-76.

34 Georges CUER, Der Französischunterricht und die französische Sprachpolitik in Deutschland nach 1945, in: Franz KNIPPING, Jacques LE RIDER (Hg.), Frankreichs Kulturpolitik in Deutschland 1945-1950, Tübingen 1987, S. 57-83. Roland LEJEUNE, La vie universitaire et scolaire. Menaces sur l'enseignement du français en Allemagne, in: Allemagne d'aujourd'hui, (1955), S. 28-35, hier S. $28 f$.

36 BaUMANN, (Anm. 33), S. 59f.

37 Ulrich LAPPENKÜPER, "Sprachlose Freundschaft«? Zur Genese des deutsch-französischen Kulturabkommens vom 23. Oktober 1954, in: Lendemains, 84 (1996), S. 67-82, hier S. 69 f.

${ }^{38}$ Deutsch-französisches Kulturabkommen vom 23. Oktober 1954, Artikel 7, in: BDFD, Bd. 1, Nr. 40, S. 184-188, hier S. 186. 
nisterpräsidenten der Länder in Düsseldorf ein "Abkommen zwischen den Ländern der Bundesrepublik zur Vereinheitlichung auf dem Gebiete des Schulwesens $«,{ }^{39}$ in welchem für die höheren Schulen mit Ausnahme der altsprachlichen Gymnasien Englisch als erste lebende Fremdsprache festgelegt wurde. Die Bundesregierung konnte den Beschluß der Länderchefs lediglich zur Kenntnis nehmen, Einflu $B$ darauf hatte sie keinen. Die französische Regierung bemühte sich in den folgenden Jahren, den Konsequenzen des Beschlusses entgegenzutreten - im großen und ganzen jedoch ohne Erfolg: Der Französischunterricht in der Bundesrepublik ging bis zum Jahr 1962 insgesamt sogar noch weiter zurück. ${ }^{40}$ Vor diesem Hintergrund war es mehr als verständlich, daß die Frage auf Drängen der französischen Regierung im Élysée-Vertrag wieder auf die politische Tagesordnung gesetzt wurde.

Im französischen Memorandum vom September 1962 war gefordert worden, daß allen Schülern der höheren Schulen in der Bundesrepublik die Möglichkeit gegeben werden solle, Französisch als erste lebende Fremdsprache zu wählen. ${ }^{41}$ Angesichts der schwierigen Rechtslage war die deutsche Reaktion vom 8. November sehr vorsichtig formuliert: «Der Vorschlag [...] wird von der Bundesregierung in Verbindung mit den Länderregierungen geprüft, die für Maßnahmen auf dem Gebiet des Erziehungswesens [...] zuständig sind « ${ }^{42}$

Diese Antwort war der Pariser Regierung viel zu vage; ${ }^{43}$ somit wurde quasi bis zur letzten Minute über die endgültige Formulierung im Vertragstext gestritten. ${ }^{44}$ Schließlich erhielt er eine sehr allgemein gehaltene Erklärung:

Die beiden Regierungen [...] werden [...] sich bemühen, konkrete Maßnahmen zu ergreifen, um die Zahl der deutschen Schüler, die Französisch lernen, und die der französischen Schüler, die Deutsch lernen, zu erhöhen. Die Bundesregierung wird in Verbindung mit den Länderregierungen, die hierfür zuständig sind, prüfen, wie es möglich ist, eine Regelung einzuführen, die es gestattet, dieses Ziel zu erreichen.

39 Archiv der Gegenwart (AdG) 1955, S. 5055f.

${ }^{40}$ Klaus-Berto von DoEmming, Deutsch in Frankreich, Französisch in Deutschland. Die Entwicklung seit 1959, in: Frankfurter Allgemeine Zeitung (FAZ), 5.7.1963.

41 MAE, Europe 1961-1970, RFA, Bd. 1598; deutsche Übersetzung: HStASt, EA 3/505, Nr. 327/1, Bl. 12 - Anlage; HStAD, Kultusministerium NW 69-314, Bl. 39.

42 HStASt, EA 3/505, Nr. 327/1, Bl. 12.

${ }^{43}$ So kritisierte François Seydoux die deutsche Antwortnote am 19.11.1962 deutlich: „Cette réponse ne va pas au-delà d'une simple manifestation de bonne volonté sur un terrain que nous considérons comme capital. " (Protokoll der Sitzung der interministeriellen Kommission am 19.11.1962; MAE, Europe 1961-1970, RFA, Bd. 1659). Auch die Kulturabteilung des französischen Außenministeriums äußerte sich am 21.11.1962 mit einer ähnlichen Stoßrichtung: "La question essentielle posée par notre mémorandum du 19 septembre était celle des rmesures à prendre pour que tout élève allemand ou français des établissements secondaires et techniques puisse choisir le français ou l'allemand comme première langue vivantec. La réponse allemande sur ce point ne nous satisfait que partiellement«; MAE, Europe 1961-1965, RFA, Bd. 1665.

44 BAUMANN (Anm. 33), S. 66-69. 
Obwohl diese endgültige Formulierung nunmehr einen eher unverbindlichen Charakter angenommen hatte und somit kaum mehr Aussagekraft besaß als die Regelung des Kulturabkommens von 1954, hoffte die französische Politik offenbar dennoch, mit Hilfe dieser Vertragsbestimmung eine Ausweitung des Französischunterrichts in der Bundesrepublik erreichen zu können. ${ }^{45}$ Der Grund für diesen Optimismus war wohl vor allem darin zu suchen, daß das "Düsseldorfer Abkommen«, welches in den Augen der politischen Akteure maßgeblich für den Rückgang des Französischunterrichts verantwortlich war, eine Laufzeit von zehn Jahren hatte und daher im nächsten Jahr eine Neuregelung anstand. ${ }^{46}$ Selbst auf deutscher Seite meldeten sich einige Politiker zu Wort, die bei der Neufassung des »Düsseldorfer Abkommens« die Stellung des Französischunterrichts verbessert sehen wollten. ${ }^{47}$ Allerdings zeichnete sich schon frühzeitig $a b, d a ß$ eine große Mehrheit der Länderregierungen für die Beibehaltung der bisherigen Regelung war, und tatsächlich unterzeichneten die Ministerpräsidenten dann im Oktober 1964 in Hamburg die »Neufassung des Abkommens zwischen den Ländern der Bundesrepublik zur Vereinheitlichung auf dem Gebiete des Schulwesens«, welche später unter der Bezeichnung "Hamburger Abkommen « bekannt wurde. ${ }^{48}$ Danach sollte die erste unterrichtete Fremdsprache win der Regel « weiterhin das Englische sein.

Der Spielraum, den diese Formulierung bot, wurde faktisch im Grunde jedoch nur im Saarland genutzt, wo Französisch seit der Eingliederung in die Bundesrepublik erste Fremdsprache war und dies auch weiterhin blieb. Im übrigen Bundesgebiet kam es dagegen insgesamt $\mathrm{zu}$ einem weiteren Rückgang des Französischunterrichts an den höheren Schulen. Im deutsch-französischen Dia$\log$ wurde in den kommenden Jahren zwar immer wieder stolz auf Statistiken verwiesen, die einen Anstieg der Schülerzahlen im Französischunterricht do-

45 So äußerte sich der Leiter der Kulturabteilung des Quai d'Otsay Jean Basdevant noch im November 1963 recht zuversichtlich: "Il nous appartient alors, non pas d'engager une bataille absurde pour éliminer l'anglais, mais de rechercher les moyens de reconquérir la place que nous estimons devoir revenir à notre langue. Pour ne parler que de l'Allemagne, c'est un des objectifs du traité de Coopération franco-allemand du 22 janvier 1963 et je suis persuadé que nous parviendrons à l'atteindre.« (Jean Basdevant, Ministre plénipotentiaire, Directeur Général des Affaires culturelles et techniques au Ministère des affaires étrangères, La Langue française dans le monde d'aujourd'hui, Conférence prononcée le 27 novembre 1963, Université des Annales, S. 8 ; PA/AA, B 90-600, Bd. 597).

$46 \S 19$ des »Düsseldorfer Abkommens«, in: AdG 1955, S. 5056.

47 Bundesfamilienminister Bruno Heck äußerte sich sogar dahingehend, daß Französisch ab 1965 generell erste Fremdsprache sein könnte; damit erntete er jedoch bei der Kultusministerkonferenz entschiedenen Widerspruch; Französisch als erste Fremdsprache, in: Augsburger Allgemeine, 26./27.1.1963.

48 Neufassung des Abkommens zwischen den Ländern der Bundesrepublik zur Vereinheitlichung auf dem Gebiet des Schulwesens vom 28.101964, in: Manfred ABELEIN (Hg.), Deutsche Kulturpolitik. Dokumente, Düsseldorf 1970, Dokument 34, S. 185-190. 
kumentierten. ${ }^{49}$ Dieser Anstieg der absoluten Zahlen beruhte aber lediglich auf dem demographischen Faktor und war keineswegs auf eine verstärkte Hinwendung zum Französischen zurückzuführen. ${ }^{\text {so }}$

Allerdings war in Frankreich eine ähnliche Entwicklung zu sehen: Hier hatte der Englisch- den Deutschunterricht schon seit dem Ersten Weltkrieg mehr und mehr verdrängt. In den 1960er Jahren war es an den französischen höheren Schulen zwar nach wie vor möglich, Deutsch als erste Fremdsprache zu wählen, jedoch machten davon nur sehr wenige Schüler Gebrauch.

In der Realität verlor die Partnersprache gegenüber dem Englischen somit in beiden Ländern an Boden, eine Entwicklung, die auch durch das 1972 unterzeichnete Abkommen zur Errichtung deutsch-französischer Gymnasien nicht aufgehalten werden konnte. Die Einführung der reformierten Oberstufe führte Ende der 1970er Jahre in Deutschland zu einem weiteren Rückgang des Französischunterrichts; ${ }^{51}$ gleichzeitig nahm auch in Frankreich das Interesse am Deutschunterricht deutlich ab. ${ }^{52}$ In den 1990er Jahren erlebte der Unterricht in der Partnersprache in beiden Ländern einen dramatischen Einbruch, so daß die Förderung des Sprachunterrichts bis heute einer der neuralgischen Punkte der deutsch-französischen Zusammenarbeit geblieben ist.

\section{Schulzeiten und Studienäquivalenzen}

Als nächstes anvisiertes Vertragsziel werden im Teil C die "Bestimmungen über die Gleichwertigkeit der Schulzeiten, der Prüfungen, der Hochschultitel und -diplome« genannt, die zweifellos ein zentrales Element der Freizügigkeit zwischen beiden Ländern und damit des Kontaktes zwischen den Bevölkerungen bilden.

Entsprechende Initiativen waren bereits in den 1950er Jahren von verschiedenen europäischen Gremien ins Leben gerufen worden, ${ }^{53}$ und auch im

So wurde beispielsweise am 21.4.1967 darauf hingewiesen, daß die Zahl der Schüler an bundesdeutschen Gymnasien mit verbindlichem Französischunterricht von 1963 (314 000) bis 1966 (343000) mum fast $10 \%$ gestiegen « sei; Kurzbericht über die Arbeitssitzung des Unterausschusses »Sprachen« der Gemischten Deutsch-Französischen Kulturkommission, Bonn, 21.4.1967, S. 2; Bayerisches Hauptstaatsarchiv (BayHStA), MK, Bd. 65915.

so Die Zahl der Schüler mit Englischunterricht stieg im gleichen Zeitraum von 443778 auf 912177 an; vgl. Amo EULER, Zur Entwicklung des Französischunterrichts seit 1945, in: Michael NERLICH (Hg.), Kritik der Frankreichforschung, Karlsruhe 1977, S. 216-233, hier S. 224. Jean-Michel HANNEQUART, Deutschunterricht in Frankreich - Ein Zustandsbericht, in: Dokumente, 59 (2003) 1, S. 28-35, hier S. 31.

53 August RUCKER, Äquivalenz der Hochschulabschlüsse im internationalen Bereich, in: Berthold MARTIN (Hg.), Jahrbuch der auswärtigen Kulturbeziehungen 1965 (2. Folge), 
deutsch-französischen Kulturabkommen von 1954 fand sich eine entsprechende Vereinbarung. ${ }^{54}$ Der im Anschluß an das Abkommen errichtete Ständige Gemischte AusschuB, in dem auch die Bundesländer vertreten waren, sollte die Arbeit weiter voranbringen. ${ }^{55}$ Im Jahr 1958 hatte dann die DeutschFranzösische Rektorenkonferenz ihre Arbeit aufgenommen, die sich in den folgenden Jahren schwerpunktmäßig mit der Erstellung von Studienäquivalenzen beschäftigte. ${ }^{56}$

Das Problem der Äquivalenzen stand also ebenfalls bereits vor der Unterzeichnung des Élysée-Vertrages auf der politischen Tagesordnung, und in dieser Frage herrschte bei den Memoranden des Jahres 1962 und bei der Ausarbeitung des Protokoll- bzw. Vertragstexts Einvernehmen zwischen der deutschen und der französischen Seite. Im Vertrag wurde schließlich festgelegt, daß »die Tätigkeit der bereits bestehenden Organe» in keiner Weise beeinträchtigt und die "zuständigen Behörden beider Staaten " gebeten werden sollten, entsprechende Bestimmungen zu erlassen. Somit wurden die Kompetenzen der Deutsch-Französischen Rektorenkonferenz und des Ständigen Gemischten Ausschusses ausdrücklich respektiert und damit indirekt auch die Zuständigkeiten der Länder und Hochschulverwaltungen auf deutscher Seite. Es kam zwar dennoch zu kleineren Streitigkeiten zwischen Bund und Ländem, ${ }^{57}$ aber die bereits etablierten Organe arbeiteten tatsächlich weiterhin effizient, so daß insbesondere die Deutsch-Französische Rektorenkonferenz bis zum Jahre 1968 eine ganze Anzahl von Äquivalenzbestimmungen für den Hochschulbereich vorlegen konnte. ${ }^{58}$ Diese waren dann jedoch mit der umfassenden französischen Hochschulreform von 1968 bereits wieder überholt!

In den folgenden Jahren war die Bildungspolitik in beiden Staaten überwiegend mit nationalen Problemen beschäftigt; zwar wurde 1971 eine DeutschFranzösische Expertenkommission für die Zusammenarbeit im Hochschul-

Bonn 1965, S. 133-143, hier S. 142; Otto SCHIEFER, Äquivalenzen im Bildungswesen, in: Berthold MARTIN (Hg.), Auswärtige Kulturbeziehungen 4, Neuwied, Berlin 1967, S. 311-318, hier S. 313.

54 Im Artikel 8 des Kulturabkommens verpflichteten sich beide Parteien, »darauf hinzuarbeiten, $\mathrm{da} B$ die in ihrem Gebiet absolvierten Studien, Prüfungswettbewerbe und Examina sowie die hierfür erlangten Zeugnisse im Gebiete des anderen Teiles entweder für Schul- oder Hochschulzwecke oder in bestimmten Fällen zur Ausübung bestimmter Berufe ganz oder teilweise als gleichwertig anerkannt wurden«; BDFD, Bd. 1, Nr. 40, S. 184-188, hier S. 186.

ss Reinhart MEYER-KaLKUS, Die akademische Mobilität zwischen Deutschland und Frankreich (1925-1992), Bonn 1994, S. 172.

56 Helmut COING, Les relations entre les universités françaises et allemandes pendant les années 50, in: Klaus MANFraSS (Hg.), Paris - Bonn: Eine dauerhafte Bindung schwieriger Partner. Beiträge zum deutsch-französischen Verhältnis in Kultur, Wirtschaft und Politik seit 1949, Sigmaringen 1984, S. 84-89, hier S. 86f.

57 BAUMANN (Anm. 9), S. 236-241.

58 Ibid. S. 244-246. 
und Wissenschaftsbereich eingesetzt und 1974 ein Hochschullehrer-Austauschprogramm begonnen, substantielle Fortschritte im Bereich der Studienäquivalenzen wurden jedoch nicht erzielt. Der Stillstand zeigte sich auch an der Tatsache, daß die deutsch-französische Rektorenkonferenz nach 1968 nicht mehr zusammentrat, und es erst wieder 1975 zu regelmäßigen Kontakten der Westdeutschen Rektorenkonferenz mit der Conférence des Présidents d'Universités kam. ${ }^{59}$

Erst am 10. Juli 1980 wurde eine grundlegende »Vereinbarung über die Befreiung von Studienzeiten, -leistungen und Prüfungen« unterzeichnet ${ }^{60}$ und bis 1986 auf die meisten Fachbereiche ausgedehnt. ${ }^{61} 23$ Jahre nach der Unterzeichnung des Élysée-Vertrages war somit zumindest ansatzweise eine Grundvoraussetzung für den akademischen Austausch geschaffen worden.

Die Bedeutung der Zusammenarbeit im Bildungsbereich wurde in den 1980er Jahren bei mehreren deutsch-französischen Konsultationstreffen herausgestellt: Nachdem bereits in der "Gemeinsamen Kulturerklärung" vom 6. Februar 1981 eine Verstärkung des Austauschs "von Lehrkräften, Forschern und Studierenden im Rahmen gesonderter Vereinbarungen gemeinsamer Arbeitsprogramme und integrierter Studiengänge ${ }^{62}$ gefordert worden war, wurde in diesem Sinne am 28. Oktober 1986 die Schaffung eines Kollegs aus deutschen und französischen Persönlichkeiten angekündigt, das Konzeptionen erarbeiten sollte, um künftig die Mobilität der Studenten und der Lehrkräfte zu erleichtern; ein längerfristiges Ziel war dabei die Einrichtung gemeinsamer Studienprogramme und integrierter Studiengänge. ${ }^{63}$ Das Gründungsabkommen für das mit dieser Aufgabe betraute Deutsch-Französische Hochschulkolleg wurde am 12. November 1987 unterzeichnet. ${ }^{64}$

Nach seiner Konstituierung legte das Hochschulkolleg zunächst die wichtigsten Kriterien für die Schaffung integrierter binationaler Studiengänge fest. Ab dem

60 Vereinbarung zwischen der Regienung der Bundesrepublik Deutschland und der Regierung der Französischen Republik über die Befreiung von Studienzeiten, -leistungen und Prüfungen zum Studium im Partnerland in den Geistes- und Naturwissenschaften, in: Sekretariat der Ständigen Konferenz der Kultusminister der Länder in der Bundesrepublik Deutschland (Hg.), Sammlung der Beschlüsse der deutsch-französischen Zusammenarbeit in den Bereichen Schule, berufliche Bildung und Hochschule auf der Grundlage des deutsch-französischen Vertrages, Bonn 1995, S. 98f.

6) Zusatzvereinbarung zur Vereinbarung vom 10.7.1980 - Studiengänge in den Fächern Wirtschaftswissenschaften (Volkswirtschaftslehre und Betriebswirtschaftslehre), Politische Wissenschaft und Rechtswissenschaften, 27.10.1986, in: ibid. S. 106f.

${ }^{63}$ Gemeinsame Erklärung über kulturelle Zusammenarbeit, 28. Oktober 1986, in: ibid. S. 12-14.

64 Deutsch-französische Vereinbarung der Außenminister über das gemeinsame Hochschulkolleg vom 12.11.1987, in: Bundesgesetzblatt 1988, Teil II, S. 137. 
Studienjahr 1990/91 wurden dann zwischen einzelnen deutschen und französischen Hochschulen die ersten derartigen Studiengänge eingerichtet, in denen deutsche und französische Studierende gemeinsam an beiden Partneruniversitäten studieren und nach erfolgreichem Studienabschluß die Diplome beider Hochschulen erwerben sollten. ${ }^{65}$ In den folgenden Jahren entstanden ungefähr 70 solcher Studiengänge, vor allem in naturwissenschaftlichen, technischen und wirtschaftswissenschaftlichen Fächern, aber auch in den Rechts-, Geistes- und Sozialwissenschaften.

Am 19. September 1997 wurde das Regierungsabkommen über die Gründung der Deutsch-Französischen Hochschule unterzeichnet, ${ }^{66}$ welche die Arbeit des Hochschulkollegs fortsetzen und weiterentwickeln sollte. Damit wurden zunächst aber nur die juristischen Grundlagen für die neuartige supranationale Hochschuleinrichtung geschaffen, in die dann ein Großteil der vom Hochschulkolleg eingerichteten Studiengänge übernommen wurde; erst im September 1999 nahm die Deutsch-Französische Hochschule in Saarbrücken dann offiziell ihre Arbeit auf. ${ }^{67}$ Sie besitzt zwar den Rechtsstatus einer Hochschule, setzt sich aber aus einem Verbund von Mitgliedshochschulen zusammen; Saarbrücken ist lediglich Sitz der Verwaltung. Die Deutsch-Französische Hochschule verfolgt seit ihrer Gründung selbstbewußt große Ziele, wie beispielsweise die Schaffung eines binationalen akademischen Grades. Dabei ist allerdings zu befürchten, daß sie Gefahr läuft, die Realitäten der Hochschulsysteme in beiden Ländern zu ignorieren. Die spektakulären Ankündigungen stehen beispielsweise in einem gewissen Widerspruch zur derzeitigen Situation in Deutschland, wo mit solchen Planungen ja zusätzlich zum Problem der Zuständigkeit der Länderregierungen die Frage der prinzipiell in der Bundesrepublik nach wie vor bestehenden Autonomie der Universitäten tangiert wird. Diese findet ja gerade im Diplomierungsrecht ihren Ausdruck - ein Recht, dessen Bedeutung im Zeichen der allseits propagierten Konkurrenz zwischen den Hochschulen sogar zunehmen soll. ${ }^{68}$ Es erscheint deswegen als sehr unwahrscheinlich, daß deutsche Universitäten auf dieses Recht zugunsten einer Verwaltungsinstitution verzichten werden! Eine weitere Schwierigkeit bildet die Beteiligung von Universitäten, Grandes Écoles und Fachhochschulen, die sich auf nationaler Ebene in einer konkurrierenden Situation befinden. Der

os Ansbert BaumanN, Grenzenlos studieren. Deutsch-französische Doppeldiplom-Studiengänge, in: Geschichte in Wissenschaft und Unterricht, 53 (2002) 9, S. 530-534.

o6 Abkommen zwischen der Regierung der Bundesrepublik Deutschland und der Regierung der Französischen Republik über die Gründung einer Deutsch-Französischen Hochschule, 19.9.1997, in: Bundesgesetzblatt 1999, Teil II, S. 451-454.

${ }^{67}$ Konrad KRAJEWSKI, Die Deutsch-Französische Hochschule - Eine Chance für die Region, in: Quoi de neuf?, 15 (1999), S. 2-5.

${ }^{68}$ Dieser Aspekt wird beispielsweise in der Diskussion um das neue Hochschulrahmengesetz in der Bundesrepublik immer wieder betont. 
Eindruck drängt sich daher auf, $\mathrm{da}$ mit der Gründung der DeutschFranzösischen Hochschule der zweite Schritt vor dem ersten gemacht wurde, gerade auch angesichts der Probleme, die viele der beteiligten Studiengänge tagtäglich erleben müssen - so gleicht die Festlegung von Studienäquivalenzen häufig nach wie vor einer Sisyphusarbeit.

Der Erfolg des Élysée-Vertrages in bezug auf die Äquivalenzen im Schulund Hochschulbereich muß also eher kritisch beurteilt werden. Die bisher erzielten Ergebnisse wären höchstwahrscheinlich auch auf der Basis der bereits bestehenden Kooperation erreichbar gewesen. ${ }^{69}$ Auch die DeutschFranzösische Hochschule, die zweifellos in Zusammenhang mit den Intentionen des Élysée-Vertrages gebracht werden kann, hat zwar die Kontakte zwischen deutschen und französischen Hochschulen deutlich voran-gebracht was zweifellos ein großes Verdienst darstellt - konkrete Fortschritte im Hinblick auf die Festlegung von Studienäquivalenzen erreichte sie jedoch kaum. Bei der unterschiedlichen Struktur der Hochschullandschaft in beiden Ländern kann die seit 1954 verfolgte Suche nach punktueller Gleichwertigkeit wohl nicht zu realistischen Lösungen führen - Titel und Diplome, die in diesen im Grunde nicht kompatiblen Ausbildungssystemen erworben wurden, lassen sich kaum in das starre Gerüst von Äquivalenzfestlegungen pressen. Erfolgversprechender wäre möglicherweise eine inhaltliche Gewichtung der Leistungsnachweise und ein System wechselseitiger Subsidiarität.

\section{Forschungskooperationen und wissenschaftliche Zusammenarbeit}

Bei der im Élysée-Vertrag geforderten Vertiefung der wissenschaftlichen Zusammenarbeit, kam ebenfalls die Unterschiedlichkeit der Hochschulsysteme und der Forschungsorganisation in beiden Ländern zum Tragen. Dennoch hatte es gerade in diesem Bereich schon vor 1963 beachtliche Initiativen gegeben: So entstanden beispielsweise ab Mitte der 1950er Jahre zahlreiche Partnerschaften zwischen deutschen und französischen Universitäten. ${ }^{70}$ Die Umwandlung des militärischen Forschungslabors im elsässischen Saint-Louis in ein binationales Forschungsinstitut im Jahr 1959 zeigte zudem, daß die Kooperation nicht allein auf den universitären Bereich beschränkt war. ${ }^{7}$ Aber schon allein an den Universitäten gab es, wie eine Umfrage der Deutschen Forschungsgemeinschaft (DFG)

So wurden bis 1968 Äquivalenzbestimmungen von der Deutsch-Französischen Rektorenkonferenz ausgearbeitet, die ja bereits vor 1963 existierte.

70 CoIng (Anm. 56), S. 84-89.

7 Rudi SCHALL, Vom Laboratoire zum Institut. Eine Chronik zur Entstehung des Instituts Saint-Louis, Saint-Louis 1988, S. 34f. 
ergab, Anfang der 1960er Jahre bereits 30 gemeinsame deutsch-französische Forschungsprojekte. ${ }^{72}$

Allerdings kam es auch in dieser Frage zunächst zu heftigen Diskussionen zwischen Bund und Ländern, ${ }^{73}$ zumal die Regierungen der Länder besonders sensibel reagierten, nachdem bei der Kabinettsumbildung im Dezember 1962 das bisherige "Bundesministerium für Atomkernenergie und Wasserwirtschaft" in ein "Bundesministerium für wissenschaftliche Forschung u umgewandelt worden war. Das Bundesministerium für wissenschaftliche Forschung mischte sich dann tatsächlich schon kurz nach der Unterzeichnung des Élysée-Vertrages aktiv in den deutsch-französischen Dialog ein: Bundesforschungsminister Hans Lenz und sein französischer Amtskollege Gaston Palewski trafen im März 1963 zusammen $^{74}$ und verabschiedeten nach Abschluß ihrer Gespräche ein gemeinsames Kommuniqué zur weiteren Entwicklung der Zusammenarbeit. ${ }^{75}$ Dies führte $\mathrm{zu}$ Verstimmungen zwischen dem Wissenschaftsministerium und dem Bevollmächtigten Kiesinger, der darin einen Eingriff in die Hoheitsrechte der Länder zu erkennen glaubte. ${ }^{76}$ Nach einigen juristischen Auseinandersetzungen verständigte man sich schließlich darauf, daß "die Begegnungen der Forschungsminister nicht in Ausführung، des deutsch-französischen Vertrages, sondern sim Geister dieses Vertrages erfolgen ${ }^{77}$ sollten. Ab 1966 waren die Ministerien für wissenschaftliche Forschung dann sogar offiziell an den deutsch-französischen Regierungstreffen beteiligt. ${ }^{78}$

Inhaltlich kann dieser Abschnitt des Élysée-Vertrages, allen Kompetenzproblemen zum Trotz, als Erfolg bewertet werden: Die wissenschaftliche Zusammenarbeit nahm in den Jahren nach 1963 einen merklichen Aufschwung. Ein besonders markantes Ergebnis der Kooperation zeichnete sich dabei schon im September $1964 \mathrm{ab}$, als sich die Fachminister beider Länder auf den gemeinsamen Bau eines Neutronenflußreaktors verständigten. ${ }^{79}$ Das Regie-

72

Claus MÜLLER-DAEHN, Die deutsch-französische Zusammenarbeit im Bereiche der wissenschaftlichen Forschung, in: Westdeutsche Rektorenkonferenz ( $\mathrm{Hg}$.), Schwarze Hefte 1962, Stücke 360-395/1962, S. 31-34, hier S. 32f.

73 BaUMANN (Anm. 9), S. 249-253.

74 Protokoll der Unterredung zwischen Bundesminister Lenz und Staatsminister Palewski vom 18.3.1963 in Paris; Bundesarchiv Koblenz (BAK), B 138, Bd. 2674.

${ }^{75}$ Gemeinsames Kommuniqué vom 19.3.1963; ibid.

76 Vgl. Ansbert BaUMANN, Kurt Georg Kiesinger - Ein Freund Frankreichs, in: Historischpolitische Mitteilungen. Archiv für Christlich-Demokratische Politik, 10 (2003), S. 225253, hier S. 236.

77 Ergebnisprotokoll der Besprechung zwischen Vertretern des Bundesforschungsministeriums und des Auswärtigen Amts am Montag, 18.11.1963; PA/AA, B 90-600, Bd. 597.

78 BAUMANN (Anm. 9), S. 252.

79 Irmgard HEINEMANN, Le traité franco-allemand du 22 janvier 1963 et sa mise en cuvre sous le général de Gaulle 1963-1969. Thèse de doctorat de spécialité (3ème cycle) „Coopération internationale«, Université de Nice, 1977, S. 333. 
rungsabkommen zur Gründung des deutsch-französischen Instituts Max von Laue - Paul Langevin (ILL) in Grenoble und dem damit verbundenen Bau des Reaktors wurde dann am 19. Januar 1967 unterzeichnet. ${ }^{80}$ Dieser modernste Neutronenflußreaktor der Welt wurde im August 1971 in Betrieb genommen. ${ }^{81}$ Bis zum heutigen Tag wird an dem Forschungsinstitut in Grenoble Spitzenforschung im Bereich der Kern- und Festkörperphysik geleistet.

Ebenfalls sehr erfolgreich verlief die Zusammenarbeit im Bereich der Fernmeldetechnik: Beide Seiten verständigten sich im April 1967 auf die Entwicklung und den Bau eines gemeinsamen Fernmeldesatelliten. ${ }^{82}$ Ein entsprechendes Abkommen wurde am 6. Juni 1967 von den beiden Forschungsministern unterzeichnet. ${ }^{83}$ Der neue Satellit "Symphonie « sollte als Kombination des französischen Kommunikationssatelliten "Saros II" mit dem deutschen Fernsehsatelliten »Olympia» der erste vielseitige europäische Fernmeldesatellit werden, der in den bis dahin allein von den Vereinigten Staaten dominierten Sektor der Weltraumforschung eindringen sollte. Tatsächlich wurde der erste entsprechende Satellit im Dezember 1974 ins All geschossen - allerdings nicht, wie ursprünglich vorgesehen, mit einer neu entwickelten europäischen Trägerrakete, sondern mit einer amerikanischen. ${ }^{84}$

In der Luft- und Raumfahrt wurden bis Ende der 1970er Jahre bedeutende Fortschritte erzielt, so daß diese bis heute eine der wichtigsten Säulen der deutsch-französischen Kooperation innerhalb der europäischen Weltraumorganisation »European Space Agency« (ESA) bildet. Im Dezember 1979 begann mit dem Start der Ariane 1 auf der Startanlage in Kourou (Französisch Guayana) die europäische Beteiligung an der internationalen Raumfahrt. ${ }^{\text {BS }}$ Auch die Entwicklung des Airbus - der Typ A 300B startete im Oktober 1972 zum Erstflug - ist ein herausragendes Beispiel der Zusammenarbeit im europäischen Rahmen ${ }^{86}$ Während sich diese Kooperation im Laufe der 1980er und

Vierter Bericht der Interministeriellen Kommission der Bundesregierung über den Stand der deutsch-französischen Zusammenarbeit, Teil II: Berichtzeitraum 1. Januar 1967 30. Juni 1967, S. 20; BAK, B 138, Bd. 2675.

81 Carlo SCHMID, Pierre-Olivier LAPIE, Die deutsch-französische Zusammenarbeit, hg. vom Presse- und Informationsamt der Bundesregierung - Stand: 20. Dezember 1972, Bonn o.J. [1973], S. 37.

82 Bulletin des Presse- und Informationsamtes der Bundesregierung Nr. 46/3.5.1967, S. 396.

83 Vierter Bericht der Interministeriellen Kommission der Bundesregierung über den Stand der deutsch-französischen Zusammenarbeit, Teil II: Berichtzeitraum 1. Januar 196730. Juni 1967, S. 20; BAK, B 138, Bd. 2675); Bulletin des Presse- und Informationsamtes der Bundesregierung Nr. 61/13.6.1967, S. 524.

${ }^{84}$ Heinz MICHAELS, Symphonie unter den Sternen, in: Die Zeit, 3.1.1975, S. 33.

85 Jürgen REICH, Ariane - ein europäisches Raketenprogramm, in: Soldat und Technik, 8 (1981), S. 424-427.

${ }^{86}$ Felix KRACHT, Der europäische Airbus: Leichtfertige Herausforderung oder marktgerechtes Denken?, o.O. o.J. [1994]. 
1990er Jahre weiter vertiefte, entstanden auch im geistes- und sozialwissenschaftlichen Bereich beachtliche Projekte. ${ }^{87}$

Auch wenn sich in vielen Forschungsbereichen die amerikanische Konkurrenz nach wie vor als übermächtig erwies, sollten die Erfolge der deutschfranzösischen Zusammenarbeit auf dem wissenschaftlichen Sektor daher insgesamt nicht zu gering veranschlagt werden.

\section{Die Gründung des Deutsch-Französischen Jugendwerks}

Die vielleicht wichtigste Maßnahme, die der dritte Teil des Élysée-Vertrages vorsah, war die Errichtung eines Austausch- und Förderungswerks für die Jugend in beiden Ländern, ausgestattet mit einem deutsch-französischen Gemeinschaftsfonds und mit einem unabhängigen Kuratorium an der Spitze.

Mit dieser Bestimmung wurde ebenfalls an vorangegangene Initiativen angeknüpft: Gleich nach Kriegsende waren von privater Seite, aber auch von der Abteilung "Jugend und Volksbildung" der französischen Militärregierung große Treffen zwischen deutschen und französischen Jugendlichen organisiert worden; ${ }^{88}$ im Kulturabkommen von 1954 wurde ein weiterer Ausbau der Jugendbegegnungen anvisiert, ${ }^{89}$ und ab Dezember $1959^{90}$ wurde das Thema zwischen beiden Regierungen wiederholt verhandelt.

De Gaulle kündigte im September 1962 gegenüber seinem Erziehungsminister an, daß er eine Intensivierung der menschlichen Kontakte zwischen Deutschen und Franzosen anstrebe, wobei der Zusammenführung der Jugend beider Völker eine Schlüsselrolle zufalle. ${ }^{91}$ Diesen Gedanken verkündete er dann bei seiner

${ }^{87}$ Hans Manfred BOCK, Der DAAD in den Deutsch-französischen Beziehungen, in: Peter ALTER (Hg.), Der DAAD in der Zeit. Geschichte, Gegenwart und zukünftigen Aufgaben, Bonn 2000, S. 196-217.

${ }^{88}$ Corine DEFRANCE, La politique culturelle de la France sur la rive gauche du Rhin 19451955, Straßburg 1994, S. 121f., S. 193-196; Stefan ZAUNER, Erziehung und Kulturmission. Frankreichs Bildungspolitik in Deutschland 1945-1949, München 1994, S. 183-200; Monique MOMBERT, Les Français et la jeunesse en Zone Française d'Occupation de 1945-1949, in: Stefan MARTENS (Hg.), Vom »Erbfeind« zum »Emeuerer«. Aspekte und Motive der französischen Deutschlandpolitik nach dem Zweiten Weltkrieg, Sigmaringen 1993, S. 175-199; Jean-Charles MOREAU, Jugendarbeit und Volksbildung in der französischen Besatzungszone, in: Jérôme VAILlANT (Hg.), Französische Kulturpolitik in Deutschland 1945-1949. Berichte und Dokumente, Konstanz 1984, S. 23-41.

89 Kulturabkommen zwischen der Regienung der Bundesrepublik Deutschland und der Regierung der Französischen Republik, Art. 5; Bundesgesetzblatt 1955, Teil II, 885-888.

90 Documents diplomatiques français (DDF) 1959II, Nr. 233/III, S. 656-665, hier S. 664.

91 Schreiben des französischen Erziehungsministers Sudreau an Außenminister Couve de Murville vom 3.9.1962 ; MAE, Europe 1961-1965, RFA, Bd. 1578 (alte Signatur). 
Ludwigsburger Rede an die Jugend ${ }^{92}$ nachdrücklich. Auf deutscher Seite stieß der französische Vorschlag auf breite Zustimmung, so daß sich die Überlegungen in den folgenden Wochen konkretisieren konnten. Ein Austausch- und Förderungswerk wurde am 15. Januar 1963 von der französischen Seite vorgeschlagen und dann erstmals in einem "Entwurf einer gemeinsamen Erklärung ${ }^{93}$ der Bundesregienung vom 16. Januar 1963 erwähnt.

Die gute Zusammenarbeit während der Ausarbeitung des Vertragstextes setzte sich auch nach der Unterzeichnung des Vertrages fort: Es wurde bald klar, daß die Gründung einer solchen binationalen Institution, für die es keine Präzedenzfälle gab, nur durch ein weiteres binationales Abkommen erfolgen könne. Die juristischen Arbeiten und die vorbereitenden Planungen über den Sitz und die Struktur der Institution kamen rasch voran, und das Gründungsabkommen $^{94}$ für das nun so genannte Deutsch-Französische Jugendwerk (DFJW) konnte somit bereits bei dem nächsten Regierungstreffen am 5. Juli 1963 unterzeichnet werden. Das wichtigste Ziel des Jugendwerks sollte ein gegenseitiges Kennenlernen, und darauf aufbauend eine künftige Verständigung, Solidarität und Zusammenarbeit sein - Ziele, die primär durch den Jugendaustausch verwirklicht werden sollten. ${ }^{95}$

Die von den Regierungen der neuen Institution zugesagte finanzielle Ausstattung in Höhe von insgesamt 40 Millionen D-Mark erweckte aber nicht nur Zustimmung; einige der bestehenden Jugendverbände befürchteten, daß sie nun mit rückläufigen finanziellen Zuschüssen zu rechnen hätten. ${ }^{96}$ Diese Besorgnis erwies sich allerdings als ungerechtfertigt: Das Jugendwerk nahm zunächst nämlich primär »eine Orientierungs- und Finanzierungsaufgabe für den Ausbau der deutsch-französischen Jugendbegegnung “ ${ }^{97}$ wahr und unterstützte somit gerade diese Organisationen. Eigene Programme wurden nur in Ausnahmefällen durchgeführt. Im übrigen verfügten die Vertreter der bestehenden Jugendverbände über die Stimmenmehrheit im Kuratorium und hatten daher einen erheblichen Einfluß auf die Entscheidungen.

Charles DE GaUlLe, Discours et messages: Pour l'effort: août 1962 - décembre 1965 , Paris 1970, S. 15-17.

93 Entwurf einer gemeinsamen Erklärung des Bundeskanzlers der Bundesrepublik Deutschland und des Präsidenten der Französischen Republik im Anschluß an die Konferenz vom 21., 22. und 23.1.1963 in Paris; MAE, Europe 1961-1970, RFA, Bd. 1598. Bundesgesetzblatt 1963, Teil II, S. 1613-1617.

$95 \mathrm{Vgl}$. Ansbert BAUMANN, Die Gründung des Deutsch-Französischen Jugendwerks: Im Spannungsfeld zwischen Bund, Ländern, Gemeinden und Zivilgesellschaft, in: Hans Manfred BOCK $(\mathrm{Hg}$.$) , Deutsch-französische Begegnung und europäischer Bürgersinn.$ Studien zum Deutsch-Französischen Jugendwerk 1963-2003, Opladen 2003, S. 39-60. Organisationen am 28. März 1963 im Auswärtigen Amt; BAK, B 106, Bd. 34564.

97 Henri MÉnUdiER, Das Deutsch-Französische Jugendwerk. Ein exemplarischer Beitrag zur Einheit Europas, Stuttgart, München 1991, S. 71. 
Die Finanzierung aus staatlichen Mitteln barg aber noch eine weitere Gefahr: Verständlicherweise versuchten möglichst viele Organisationen eine finanzielle Unterstützung zu erhalten, und der Jugendaustausch drohte so zu einer Massenveranstaltung zu verkommen, bei der die Quantität der Kontakte weit mehr zählte als deren Qualität. Diese Entwicklung wurde schon im Mai 1964 in einem Artikel in der Süddeutschen Zeitung ${ }^{98}$ beklagt, der innerhalb der Bundesregierung für erhebliche Unruhe sorgte. Das Bundeskanzleramt bat das Bundesministerium für Familien und Jugendfragen unverzüglich um eine Stellungnahme zu den Vorwürfen, ${ }^{99}$ und die Antwort ${ }^{100}$ aus dem Ministerium wurde im Kanzleramt eher reserviert zur Kenntnis genommen: "Aus dem sehr allgemein und in verharmlosendem Ton gehaltenen Antwortschreiben des Bundesfamilienministeriums ist erkennbar, daß die Arbeit des DeutschFranzösischen Jugendwerks noch nicht in allen Punkten reibungslos und mit dem erwünschten Erfolg abläuft«. ${ }^{101}$ In der Tat war das Jugendwerk anfangs dem großen Echo, das es ausgelöst hatte, kaum gewachsen. Die Zahl der Förderanträge überschritt 1964 bereits im Sommer den Etat für das gesamte Tätigkeitsjahr in Höhe von 40 Millionen D-Mark, ${ }^{102}$ so daß das Kuratorium sämtliche privaten oder öffentlichen Institutionen in der Bundesrepublik Deutschland und in Frankreich auffordern mußte, auch genügend eigene Mittel für ihre Austauschprojekte zur Verfügung zu stellen. ${ }^{103}$

Dennoch waren schon allein die Zahlen der Austauschaktivitäten beeindrukkend: Das Jugendwerk förderte im Jahr 1964 in Frankreich 2979 und in Deutschland 2246 Programme $^{104}$ und ließ dabei 97297 jungen Franzosen und 120336 jungen Deutschen eine finanzielle Unterstützung zukommen. ${ }^{105} \mathrm{Au}-$ Berdem bemühten sich die Verantwortlichen des Jugendwerks in der Folgezeit um eine qualitative Verbesserung der Austauschprogramme, ${ }^{106}$ so daß sich diese gerade in Zeiten der politischen Abkühlung im deutsch-französischen

98 Alfred FRISCH, Deutsch-französisches Jugendwerk in Gefahr. Die gute Sache erstickt an der Verwaltungsroutine. Arbeit des Goethe-Instituts in Frankreich eingeschränkt, in: Süddeutsche Zeitung, 13.5.1964.

99 Staatssekretär Globke an das Bundesministerium für Familien- und Jugendfragen, 14.5.1964; BAK, B 136, Bd. 5531.

${ }^{100}$ Bundesminister für Familie und Jugend (i.A. Ludwig) an Staatsekretär Globke, 29.5.1964; ibid.

101 Vermerk des Referats 7 im Bundeskanzleramt vom 8.6.1964; ibid.

${ }^{102}$ Bericht der Interministeriellen Kommission der Bundesregierung über den Stand der deutsch-französischen Zusammenarbeit vom 1.6.1964, S. 15; BAK, B 138, Bd. 2674.

${ }^{103}$ Ergebnisniederschrift der vierten Sitzung des Kuratoriums des Deutsch-Französischen Jugendwerks; PA/AA, B 90-600, Bd. 412.

${ }^{104}$ DFJW/OFAJ, Tätigkeitsbericht / Rapport d'Activité 1963-1968, Bad Honnef ${ }^{2} 1969$, S. 10.

${ }^{105}$ Ibid. S. 23.

${ }^{106}$ Vermerk des Bundeskanzleramts, 18.5.1965; BAK, B 136, Bd. 5531. 
Verhältnis sehr erfreulich entwickelten. Selbst das Bulletin des Bundespresseamts konstatierte deswegen im September 1965: "In der sog. shohen Politik hat es, was das deutsch-französische Verhältnis betrifft, seit der Unterzeichnung des Freundschaftsvertrages schon manche Komplikation und manche Enttäuschung gegeben. Das Deutsch-Französische Jugendwerk jedoch bildet eine rühmliche Ausnahme ${ }^{107}$ Auch auf französischer Seite wurde in den Halbjahresberichten der Kulturabteilung des Quai d'Orsay die erfreuliche Arbeit des Jugendwerks als ein Lichtblick in den belasteten Beziehungen herausgestellt. $^{108}$

In den ersten Jahren konnte das Jugendwerk seine Tätigkeit unter gesicherten finanziellen Verhältnissen ausüben; nach der Finanzkrise im Jahr 1968 kürzte die französische Regierung allerdings ihren Anteil am Gesamtetat für das Haushaltsjahr 1969 von 25 Millionen Francs auf 23,5 Millionen Francs, umgerechnet also von 20 Millionen D-Mark auf 18,8 Millionen D-Mark. ${ }^{109}$ Die Bundesregierung steuerte sogar nur noch 18 Millionen D-Mark ${ }^{110}$ bei. In den darauffolgenden Jahren wurden die staatlichen Mittel noch weiter zurückgefahren, was auch die Handlungsspielräume der Organisation mehr und mehr einengte. Der dem Jugendwerk 1963 zugestandene Gesamtetat in Höhe von 40 Millionen D-Mark wurde erst wieder im Jahre 1991 erreicht. ${ }^{1 \prime \prime}$

Trotz dieser Schwierigkeiten setzte das DFJW den intensivsten Jugendaustausch in Gang, den es je zwischen zwei Ländern gab: Bis heute nahmen mehr als 7 Millionen Jugendliche an über 220000 Programmen teil. Bemerkenswert dabei ist, daß von Anfang an darauf geachtet wurde, die Begegnungen auch für solche Jugendliche offen zu halten, die in der Regel kaum die Möglichkeit zu Auslandsaufenthalten haben - so wurden beispielsweise jugendliche Arbeitnehmer in besonderer Weise an den Austauschmaßnahmen beteiligt.

Die Aktivitäten des Jugendwerks beschränkten sich zudem nicht ausschließlich auf die "Jugend«; so wurden in den ersten Jahren nach seiner Gründung besonders Städtepartnerschaften und die meistens darin eingebundenen Schulpartnerschaften gefördert: Deutsche und französische Städte, die eine Partnerschaft eingehen wollten, wurden durch beträchtliche finanzielle Zuschüsse unterstützt. ${ }^{112}$ Mit großem Erfolg: Die Zahl der Neugründungen

${ }^{107}$ Bulletin Nr. 153/15.9.1965, S. 1238.

${ }^{108}$ État des relations culturelles entre la France et la République fédérale d'Allemagne, 14.1.1965; MAE, Europe 1961-1970, RFA, Bd. 1476; 27.1.1966; MAE, Europe 19611970, RFA, Bd. 1477; 2.7.1966; ibid.

${ }^{109}$ MÉNUdier (Anm. 94), S. 123.

${ }^{110}$ Ibid. S. 125.

111 Ibid.

112 DFJW/OFAJ, Tätigkeitsbericht/Rapport d'Activité 1963-1973, Paris 1974, S. 77. 
von deutsch-französischen Städtepartnerschaften stieg im Jahre 1963 sprunghaft an und blieb bis 1975 auf einem anhaltend hohen Niveau. ${ }^{113}$

In solchen indirekten Einflüssen liegt möglicherweise eine wesentliche Bedeutung des Élysée-Vertrages, der gerade in den Erziehungs- und Jugendfragen auf eine Popularisierung der deutsch-französischen Beziehungen abzielte, die, in einer langfristigen Perspektive, das Verhältnis der beiden Völker zueinander positiv beeinflussen sollte. Auch wenn sich die Wirkungen dieser Bestimmungen nur schwer in gängigen Kategorien messen lassen, sollte doch der Versuch gewürdigt werden, ein Beziehungsgeflecht zwischen dem deutschen und dem französischen Volk zu etablieren, das letztlich unabhängig von diplomatisch-politischen Imponderabilien sein sollte!

${ }^{113}$ Thomas GRUNERT, Langzeitwirkungen von Städte-Partnerschaften. Ein Beitrag zur europäischen Integration, Kehl, Straßburg 1981, S. 99. 